\title{
Surfactant Sputtering: Theory of a new method of surface nanostructuring by ion beams
}

\author{
R. Kree ${ }^{\mathrm{a}}$, T. Yasseri ${ }^{\mathrm{a}}$, A.K. Hartmann ${ }^{\mathrm{b}}$ \\ ${ }^{a}$ Institut. f. Theoretische Physik, Friedrich-Hund-Platz 1, Georg-August University Goettingen, D-37077 Goettingen, Germany \\ ${ }^{b}$ Institut f. Physik, Universitaet Oldenburg, 26111 Oldenburg, Germany
}

\begin{abstract}
We present a new Monte Carlo model and a new continuum theory of surface pattern formation due to "surfactant sputtering", i.e. erosion by ion-beam sputtering including a submonolayer coverage of additional, cosputtered surfactant atoms. This setup, which has been realized in recent experiments in a controlled way leads to a number of interesting possibilities to modifiy pattern forming processing conditions. We will present three simple scenarios, which illustrate some potential applications of the method. In all three cases, simple Bradley-Harper type ripples appear in the absence of surfactant, whereas new, interesting structures emerge during surfactant sputtering.
\end{abstract}

Key words:

\section{Introduction}

The self-organized evolution of surface patterns induced by ion beam sputtering (IBS) has been intensively studied, both experimentally and theoretically, under various material and processing conditions (see Ref. [1] for a recent review). The variety and quality of the emerging nanoscaled ripples or quantum dot structures indicate that IBS may become an efficient tool for fabricating devices of nanotechnologies. Therefore, increasing experimental effort is spent on extending and improving the control of processing conditions leading to taylor-made, high quality surface structures [2]. Up to now, a wide variety of crystalline and amorphous target materials and of processing conditions, including ion species, ion energy, temperature, angle of ion beam incidence and ion beam profile have been used to control, vary and optimize surface structures .

Recently [3] a novel sputter erosion technique has added further possibilities of fine-tuning processing conditions in many ways. It prepares a submonolayer coverage of the substrate (A) surface with "surfactant (B) atoms", which are constantly redeposited by cosputtering of a nearby surfactant target. The current $\mathbf{J}_{B+}$ maintaining the average coverage during sputter erosion, is tuned beyond the resputtering limit, so that film growth of B is suppressed. Depending on diffusion, mixing and alloying properties of surfactant and target Preprint submitted to Nuclear Instruments and Methods atoms, the surfactant distribution may tend either to a homogeneously mixed A-B layer, or it may develop inhomogeneous patterns by mechanisms like surface segregation, island formation, clustering, diffusion instabilities or attachment to surface defects. The coverage by a surfactant density significantly changes the local sputtering yield of the substrate. In most cases the yield is reduced, in exceptional cases it may also be increased [4]. These local changes provide a feed-back mechanism between the pattern formation processes of substrate and of surfactant atoms. By choosing appropriate surfactant-substrate combinations, a variety of surface patterns may be obtained in a controlled way [3]. Furthermore, the surfactant distribution may itself become a technologically useful, self-organized structure, like an ordered array of dots or nanowires. The technique also allows to prepare spatial profiles of $\mathbf{J}_{B+}$, so that modifications of the yield from nanometer to macroscopic length scales can be controlled.

We have set up both a Monte Carlo simulation model and a continuum theory of surfactant sputtering. Here, we will only consider the special case of demixing surfactant-substrate combinations and assume that surfactant atoms are always on top of the surface provided by the substrate. In the following we present the derivations of our models and some results obtained for three prototypical scenarios, which serve to illustrate potential applications of surfactant sputtering.

January 28, 2009 


\section{Continuum theory and Monte Carlo model}

The Monte Carlo model is based on the algorithm presented in Ref. [5, 6, 7, 8]. It uses a a discrete height profile $h(x, y)$ of a solid-on-solid surface over a $L \times L$ $(x, y)$-lattice. Each surface site is occupied either by a substrate atom (A) or by a surfactant atom (B). Random ion paths in direction $-\mathbf{d}$ (inclined by $\theta$ with respect to the z-axis) are traced until they have traversed a length $d$ in the substrate and are stopped at $\mathbf{r}_{o}$. A surface atom at $\mathbf{r}=(x, y, h)$ is eroded with a probability proportional to the deposited energy $E$ arising from the stopped ion. The spreading of energy is described by an anisotropic Gaussian [9] $E\left(\mathbf{r}=\mathbf{r}_{s}-\mathbf{r}_{0}\right)=C \exp \left(-r_{\|}^{2} / 2 a_{\|}^{2}-r_{\perp} / 2 a_{\perp}^{2}\right)$, where $r_{\|}^{2}=(\mathbf{r} \cdot \mathbf{d})^{2}$ and $r_{\perp}^{2}=r^{2}-(\mathbf{r} \cdot \mathbf{d})^{2}$. The sputtering probabilities for $\mathrm{A}$ and $\mathrm{B}$ atoms may be different, but note that only surface atoms are sputtered off. Thus a $\mathrm{B}$ atom at $\mathbf{r}$ suppresses the A sputter yield at this site completely. In addition, a B atom may also reduce the sputter yield of $\mathrm{A}$ atoms at a nearest neighbor site by a factor $1-\Lambda, 0 \leq \Lambda \leq 1$. B atoms, which are sputtered off are immediately replaced via random redeposition. In the present work, we only consider B atoms on the surface (i.e. we exclude B atoms in the A bulk) and restrict the thickness of the B-layer to at most 1 . This is achieved by forbidding any moves of atoms, which end up on top of a B atom. All atoms perform thermally activated hops to unoccupied nearest neighbor sites with Arrhenius rates $k_{a}=k_{0 a} \exp \left(-\beta E_{a}(i \rightarrow f)\right),(a=A, B)$, where $\beta=1 / k T$ and $E_{a}(i \rightarrow f)$ denotes the barrier for a hop of an "a" particle from initial site i to final site $f$. This barrier consists of two contributions: a term, which counts the net number of bonds, which have to be broken by the move and an Ehrlich-Schwoebel (ES) barrier [10]. The former is given by $E_{b}(i \rightarrow f)=E_{0 a}+E_{N N}$, where $E_{N N}=\left(n_{A}(i)-n_{A}(f)\right) E_{a A}+\left(n_{B}(i)-n_{B}(f)\right) E_{a B}$, if it is positive, whereas it is zero otherwise. The barrier $E_{0 a}$ sets the hopping rate on a flat surface. $n_{a}(i)$ is the number of in-plane nearest neighbors of type "a" and $E_{a b}$ is the energy neccessary to break an $a b$-bond. The Ehrlich-Schwoebel barrier seen by an "a" atom equals the constant $E_{E S a}$, if $\mathrm{f}$ and $\mathrm{i}$ are in plane and $\mathrm{f}$ is at the upper edge of a step on the surface, otherwise it is zero, thus it prevents the approach of atoms towards a downhill step edge on a terrace. We absorb the factor $G_{a}=\exp \left(-\beta E_{0 a}\right)$ arising from substrate bonds into a rescaled attempt rate $k_{1 a}=k_{0 a} G_{a}$. The time scales are calibrated by the incident ion flux $F$ (in ions per surface atoms of a flat surce) and by $k_{1 a}$, such that there are $k_{1 a}$ diffusion step trials (for every surface atom) every $F L^{2}$ erosion steps in an $L \times L$ system. Typical default values used in our simulations are: $E_{0 a}=0.75 \mathrm{eV}, E_{A A}=0.18$
$\mathrm{eV}, E_{E S}=0.15 \mathrm{eV}$. $E_{A B}$ is varied from $0 \cdots E_{A A}$ and $E_{B B}$ from $E_{A A} \cdots 0.6 \mathrm{eV}$. We have studied B yields from $1 \cdots 10$ times the A yield

The continuum description is based on the same physical assumptions. Its derivation starts from the balance equation of mass within a subvolume $V$ of the A substrate and its surface $\partial V$. The bulk density $\rho$ of the substrate is assumed to be constant, the surfactant is characterized by a varying surface density $\sigma$. Here, we neglect an excess surface density of A adatoms, which will be taken into account in forthcoming work. If we denote the erosion velocity (normal to the surface) by $v_{n}$, the balance of substrate mass is expressed as $\rho v_{n}=-J_{A}-\operatorname{div}_{S} \cdot \mathbf{j}_{\mathbf{A}}$ with erosion current $J_{A}$ and surface diffusion current density $\mathbf{j}_{\mathbf{A}} \cdot \operatorname{div}_{S}$ denotes the surface divergence. The balance equation for $\sigma$ takes on the form

$$
\frac{D_{S} \sigma}{d t}=-J_{B}+\mathbf{J}_{r d} \cdot \mathbf{n}-\operatorname{div}_{S} \mathbf{j}_{B}
$$

Here $\mathbf{n}$ denotes the outward normal unit vector of the surface. The transport derivative, based upon the results in Ref.[11],

$$
D_{S} \sigma / d t=\partial_{t} \sigma+v_{n} \mathbf{n} \cdot \nabla \sigma-\sigma v_{n} K,
$$

has not yet been used in continuum theories of ion beam sputtering. It takes into account all the temporal changes of the surface morphology. Here, $\kappa=\operatorname{div} \mathbf{n}$ denotes the mean curvature of the surface.

The model is completed by expressing the currents and current densities in terms of the surface geometry and the surfactant density. Expressions for the erosion currents in the absence of surfactants are available from the theory of Bradley and Harper [12] and its generalizations $[13,14,15]$. In addition, we take into account modifications of the sputtering yields so that the erosion currents of substrate and surfactant, $J_{A}$ and $J_{B}$, respectively, are given by $J_{A}=J_{0} Y_{A} g_{A}(\sigma)\left(1-v_{B H}\right)$ and $J_{B}=J_{0} Y_{B} g_{B}(\sigma)\left(1-v_{B H}\right) . J_{0}$ denotes the flux of incident ions, $Y_{A}, Y_{B}$ are the sputtering yields of the pure A and B system, respectively. We keep the first terms of the standard gradient expansion of the yield modification due to surface morphology $[12,15]$,

$$
v_{B H}=\mathbf{w} \cdot \nabla h+a_{x} \frac{\partial^{2} h}{\partial x^{2}}+a_{y} \frac{\partial^{2} h}{\partial y^{2}}+b_{x}\left(\frac{\partial h}{\partial x}\right)^{2}+b_{y}\left(\frac{\partial h}{\partial y}\right)^{2} .
$$

The factors $g_{A}, g_{B}$ are taken to be of the form $g_{A}(\sigma)=$ $\max (1-\lambda \sigma, 0)$ and $g_{B}(\sigma)=\sigma$ to parametrize the changes of sputtering yields due to small B coverages. This choice is in accordance with the experimental findings of [3] for small surfactant densities. Note that the 
nonlinearities in $v_{B H}$ may be kept up to any desired order, but we have to keep the full geometrical nonlinearities in the transport derivatives, because otherwise we would violate mass conservation during surface diffusion.

The surface diffusion current densities $\mathbf{j}_{\mathbf{A}}$ and $\mathbf{j}_{\mathbf{B}}$ contain near-equilibrium terms, which are driven by the reduction of surface free energy and non-equilibrium terms, which are driven by the external erosion and redeposition fluxes. Extensive discussions of these terms are available in the literature on solid surface growth (see, for example [16]). Here, we only take into account simple contributions arising from expansions in $\nabla h$ and $\sigma$ and a simple, phenomenological expression for the non-equilibrium Ehrlich-Schwoebel (ES) current, so that for the simplest case of isotropic (amorphous) samples

$$
\operatorname{div}_{S} \mathbf{j}_{\mathbf{A}} \approx K_{1}\left(\nabla^{2}\right)^{2} h+K_{A, E S} \nabla^{2} h\left(1-l_{E S}^{2}(\nabla h)^{2}\right)
$$

and

$$
\operatorname{div}_{S} \mathbf{j}_{\mathbf{B}} \approx-\nabla\left(D_{B} \nabla \sigma\right)+K_{B, E S} \sigma \nabla^{2} h\left(1-l_{E S}^{2}(\nabla h)^{2}\right)
$$

Continuum models, which also consider the time evolution of densities in a surface layer, have appeared in the literature, which differ in important aspects from the present work. In ref [17] a bulk binary alloy is considered, and in refs. [18] and [19] a layer of adatoms of the target material is included. Both papers also differ from the present work in the physical concepts, which underly the evolution equation of the surface density.

In the numerical solutions presented below, we have extended this simplest diffusion model in two ways: (i) we explicitely took into account a (cubic) anisotropy arising from eroding a (100) surface and (ii) we allowed for clustering of the $\mathrm{B}$ atoms by putting $D_{B}=$ $D_{B}^{0} \max (1-c \sigma, 0)$. Details will be presented elsewhere. This has been done to facilitate comparisons with our Monte Carlo simulations, which naturally include these effects. The system of non-linear partial differential equations, which make up the continuum model have been soved by a finite element method using ideas from [20]

The continuum and the MC approach are related in the sense that both are built upon the same basic physical mechanisms of erosion and surface diffusion. The description of erosion is in both models based upon Sigmund's sputtering formula and the continuum theory contains a large lengthscale description of the surface diffusion of substrate and surfactant as implemented in the MC model [5].

\section{Three scenarios of surfactant sputtering}

We now present three simple scenarios of IBS pattern formation in the presence of a co-sputtered surfactant.In all cases usual Bradley-Harper type ripples will develop in the absence of surfactant.

In the first example, depicted in Fig. 1 we demonstrate that the ripple pattern modulates the surface density of those surfactants, which do not significantly change the sputter yield of the substrate. The surfactant diffusion parameters chosen in Fig. 1 would lead to Bclustering on a flat surface. On the rippled surface, the surfactant accumulates on the crests of ripples, where it tends to cluster. Note that such droplets of surfactant can lead to pronounced shadowing effects, which are not included in the continuum theory. A similar scenario has been found experimentally on Si surfaces, using $\mathrm{Ag}$ as surfactant. In fact, one expects a generic uphill current of any surfactant, which suppresses the yield, because it is sputtered preferrably from valleys (Bradley Harper mechanism) but redeposited randomly. Thus material is moved out of valleys.

The second scenario, shown in Fig. 2, demonstrates, that a strong suppression of the substrate sputter yield due to surfactant coverage may lead to very smooth surfaces, reminescent of layer-by-layer erosion, instead of rippled topographies. In fact, we started the numerical solution with an initially rough surface and observed a monotonous decrease of surface roughness.

The third scenario, depicted in Fig. 3 demonstrates, how dot-like patterns, due to Ehrlich-Schwoebel diffusion, can be generated by a surfactant. Without surfactant, the growth of ripples is the dominant process and typical Bradley-Harper ripples emerge, even in the presence of Ehrlich-Schwoebel diffusion. Coverage with a surfactant tends to suppress the Bradley-Harper instability and Ehrlich-Schwoebel diffusion can become the dominant, pattern-forming mechanism. Note that the ES-type dots can form an ordered array. Ordering is supported, if preliminary ripple structures break up into dots. Ordered dots are more clearly visible in the continuum theory. The MC dynamics has been limited to the erosion of 5 monolayers to keep it consistent with the calibrated time in the continuum theory, but the effects of noise are too strong to detect dot ordering in MC within this time interval.

In this scenario, the distribution of surfactant still depends upon its diffusion and clustering properties.

\section{References}

[1] W.-L. Chan and E. Chason. Making waves: Kinetic processes controlling surface evolution during low energy ion sputtering. 
Journal of Applied Physics, 101(12):121301, 2007.

[2] F. Frost, B. Ziberi, A. Schindler, and B. Rauschenbach. Surface engineering with ion beams: from self-organized nanostructures to ultra-smooth surfaces. Appl. Phys. A, 91:551-559, 2008.

[3] H. Hofsäss and K. Zhang. Surfactant sputtering. Appl. Phys. A, 92(3):517-524, Aug 2008.

[4] S. Berg, A. Barklund, B. Gelin, C. Nender, and I. Katardjiev. Atom assisted sputtering yield amplification. J. Vac. Sci. Technol, A10:1592, 1992.

[5] A. K. Hartmann, R. Kree, U. Geyer, and M. Kölbel. Long-time effects in a simulation model of sputter erosion. Phys. Rev. B, 65(19):193403, May 2002.

[6] E. O. Yewande, A. K. Hartmann, and R. Kree. Propagation of ripples in monte carlo models of sputter-induced surface morphology. Physical Review B (Condensed Matter and Materials Physics), 71(19):195405, 2005.

[7] E. O. Yewande, R. Kree, and A. K. Hartmann. Morphological regions and oblique-incidence dot formation in a model of surface sputtering. Physical Review B (Condensed Matter and Materials Physics), 73(11):115434, 2006.

[8] E. O. Yewande, R. Kree, and A. K. Hartmann. Numerical analysis of quantum dots on off-normal incidence ion sputtered surfaces. Physical Review B (Condensed Matter and Materials Physics), 75(15):155325, 2007.

[9] P. Sigmund. Theory of sputtering. i. sputtering yield of amorphous and polycrystalline targets. Phys. Rev., 184(2):383-416, Aug 1969.

[10] M. Bielh. Lattice gas models and Kinetic Monte Carlo simulations of epitaxial growth. Birkuser, 2004.

[11] P. Cermelli, E. Fried, and M.E. Gurtin. Tranport relations for surface integrals arising in the formulation of balance laws for evolving fluid interfaces. J. Fluid. Mech., 544:339-351, 2005.

[12] R. M. Bradley and J. M. E. Harper. Theory of ripple topography induced by ion-bombardment. J. Vac. Sci. Technol. A, 6(4):2390-2395, Jul 1988.

[13] R. Cuerno and A.-L. Barabási. Dynamic scaling of ion-sputtered surfaces. Phys. Rev. Lett., 74(23):4746-4749, Jun 1995.

[14] G. Carter. Effect of surface-height derivative processes on ion-bombardment-induced ripple formation. Phys. Rev. B, 59(3):1669-1672, Jan 1999.

[15] M. A. Makeev, R. Cuerno, and A. L. Barabási. Morphology of ion-sputtered surfaces. Nucl. Instr. and Meth. in Phys. Res. B, 197(3-4):185-227, Dec. 2002.

[16] T. Michely and J. Krug. Islands, Mounds and Atoms - Patterns and Processes in Crystal Growth Far From Equilibrium. Springer, Berlin, 2004.

[17] V. B. Shenoy, W. L. Chan, and E. Chason. Compositionally modulated ripples induced by sputtering of alloy surfaces. Physical Review Letters, 98(25):256101, 2007.

[18] T. Aste and U. Valbusa. Ripples and ripples: from sandy deserts to ion-sputtered surfaces. New Journal of Physics, 7:22, 2005.

[19] M. Castro, R. Cuerno, L. Vázquez, and R. Gago. Self-organized ordering of nanostructures produced by ion-beam sputtering. Physical Review Letters, 94(1):016102, 2005.

[20] M. Burger. Surface diffusion including adatoms. Commun Math. Sci, 4(1):1-51, 2006.

\section{Figure captions:}

Fig.(1): Lower panels: Ripple pattern of a substrate with 0.4 monolayer coverage of surfactant with $Y_{A}=Y_{B}$ and strong clustering after 5 ions per surface atom, Left: MC simulation $E_{A B}=0, E_{B B}=0.6 \mathrm{eV}$, Right panel: Continuum theory $(c=2)$. The direction of the ion beam is inclined by $\phi=0.2$ with respect to the $\mathrm{x}$-axis. No Ehrlich-Schwoebel effects are included. The bars mark identical regions in upper and lower panels, respectively. Upper panels: Corresponding distributions of surfactant.

Fig. (2):Upper panels: BH-type ripples without surfactant, left: MC simulation, right: Continuum theory. Lower panels: Strong suppression of sputter yield of substrate by surfactant leads to smooth surfaces left: $\Lambda=0.25$ in MC. right: $\lambda=2$ in continuum theory, here, the height scale is enhanced by a factor 100 with respect to the corresponding upper panel

Fig. (3):Upper panels: ES diffusion of substrate and surfactant is chosen such that BH instability dominates. No suppression of substrate sputter yield by surfactant. Lower panels: Moderate suppression of substrate sputter yield by surfactant $(\Lambda=0.1$ in $\mathrm{MC}, \lambda=0.6$ in continuum)tends to weaken the $\mathrm{BH}$ instability, so that ES diffusion can influence the pattern formation. Left panels: MC simulation, Right panels: Continuum theory 

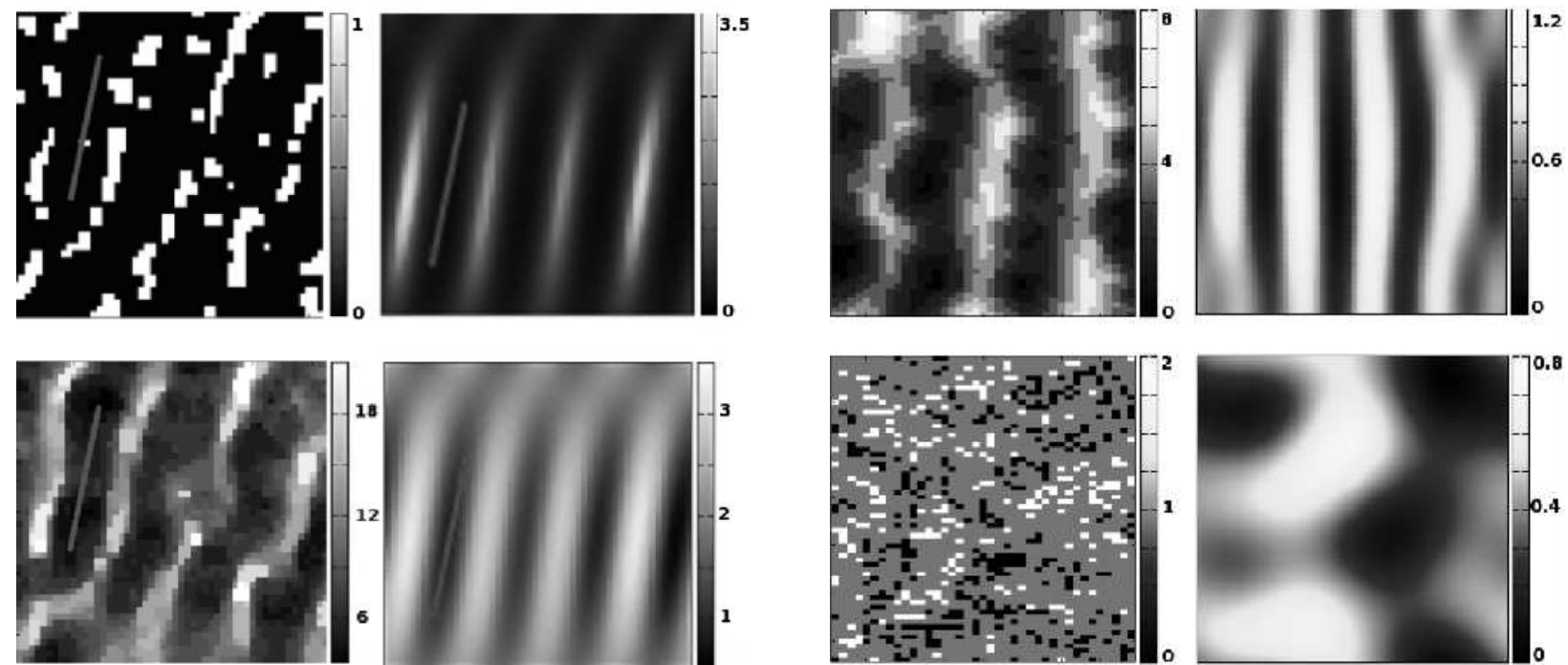

Figure 1: Lower panels: Ripple pattern ....

Figure 2: Upper panels: BH-type ripples .... 

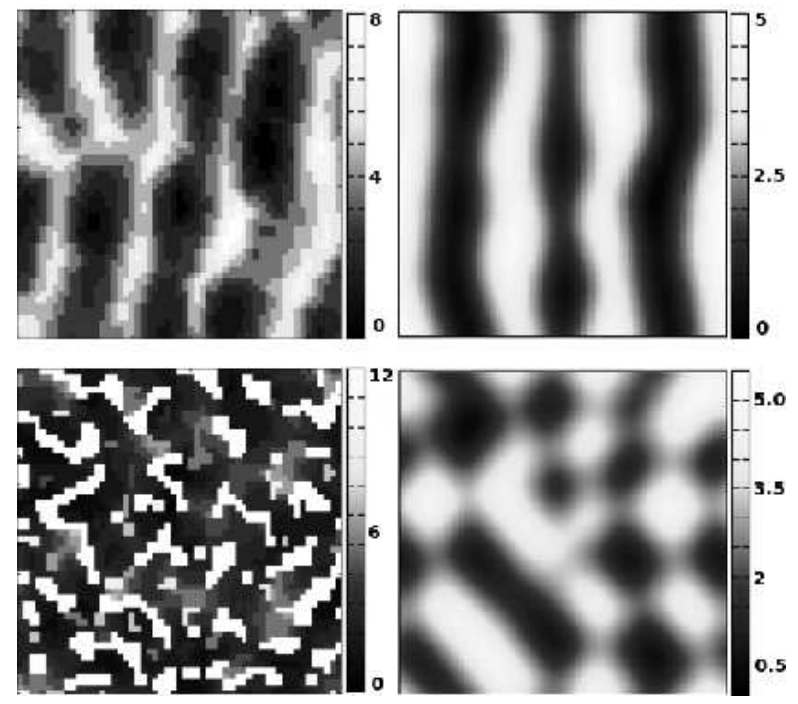

Figure 3: Upper panels: ES diffusion of ..... 